\title{
Revista Colombiana de

\section{Lecitina colesterol aciltransferasa en mujeres postmenopáusicas y su relación con factores bioquímicos y antropométricos asociados a riesgo cardiovascular}

\author{
Luz A. Sarmiento-Rubiano*, Eduin J. Acosta, Alfonso Suárez-Rodríguez, \\ Lucía P. Sánchez-Majana, Roberto C. Rebolledo-Cobos y Jimmy E. Becerra
}

Grupo de Investigación Alimentación y Comportamiento Humano, Universidad Metropolitana, Fundación Hospital Universitario Metropolitano, Barranquilla, Colombia

Recibido el 7 de marzo de 2018; aceptado el 15 de mayo de 2019

Disponible en Internet el 28 de octubre de 2019

\author{
PALABRAS CLAVE \\ Lecitina colesterol \\ aciltransferasa; \\ Postmenopausia; \\ Dislipidemia; \\ Arteriosclerosis; \\ Enfermedades \\ cardiovasculares
}

\begin{abstract}
Resumen
Objetivo: determinar los valores séricos de la enzima lecitina colesterol aciltransferasa en un grupo de mujeres postmenopáusicas, y establecer su relación con factores asociados a riesgo cardiovascular.

Materiales y métodos: estudio descriptivo transversal prospectivo, correlacional, que incluyó 56 mujeres postmenopáusicas en quienes se evaluaron variables antropométricas y bioquímicas (perfil lipídico y glicemia basal) asociadas a riesgo cardiovascular y se correlacionaron con las concentraciones séricas de lecitina colesterol aciltransferasa.

Resultados: los valores séricos promedio de dicha enzima fueron 7,89 $\pm 1,26 \mu \mathrm{g} / \mathrm{ml}$. Las mujeres con valores de índice de masa corporal superior a 25 tienen niveles séricos de lecitina colesterol aciltransferasa significativamente mayores que aquellas que tienen índice de masa corporal normal. No se observaron relaciones significativas entre los niveles de lecitina colesterol aciltransferasa y las variables bioquímicas evaluadas.

Conclusiones: este trabajo es uno de los primeros que evalúa los niveles séricos de lecitina colesterol aciltransferasa en mujeres postmenopáusicas del Caribe colombiano. Se encontró una relación significativa entre los niveles séricos de lecitina colesterol aciltransferasa y los valores de índice de masa corporal elevados. Se requieren nuevos estudios para entender mejor la relación entre los niveles séricos de lecitina colesterol aciltransferasa y el riesgo cardiovascular en mujeres postmenopáusicas.

(C) 2019 Sociedad Colombiana de Cardiología y Cirugía Cardiovascular. Publicado por Elsevier España, S.L.U. Este es un artículo Open Access bajo la licencia CC BY-NC-ND (http:// creativecommons.org/licenses/by-nc-nd/4.0/).
\end{abstract}

\footnotetext{
* Autor para correspondencia.

Correo electrónico: lusarru@hotmail.com (L.A. Sarmiento-Rubiano).
} 


\section{KEYWORDS}

Lecithin cholesterol

acyltransferase;

Postmenopause;

Dislipidaemia;

Arteriosclerosis;

Cardiovascular

diseases

\section{Lecithin cholesterol acyltransferase in serum of postmenopausal women in relation to biochemical and anthropometric factors associated to cardiovascular risk}

\begin{abstract}
Objective: To determine the serum levels of lecithin cholesterol acyltransferase in a group of postmenopausal women and to establish their relationship with factors associated with cardiovascular risk.

Materials and methods: A descriptive, correlational and cross-sectional study was performed that included 56 postmenopausal women. Anthropometric and biochemical (lipid profile and baseline blood glucose) variables associated with cardiovascular risk were measured, and were correlated with the serum concentrations of lecithin cholesterol acyltransferase.

Results: The mean serum level of lecithin cholesterol acyltransferase was $7.89 \pm 1.26 \mu \mathrm{g} / \mathrm{ml}$. The women with a body mass index greater than 25 had significantly higher serum levels of the enzyme than those that had a normal body mass index. No significant relationships were observed between the levels of lecithin cholesterol acyltransferase and the biochemical variables evaluated.

Conclusions: This study is one of the first that has evaluated the serum levels of lecithin cholesterol acyltransferase in postmenopausal women of the Colombian Caribbean. A significant relationship was found between the serum levels of lecithin cholesterol acyltransferase and elevated values of the body mass index. Further studies are required for a better understanding of the relationship between the serum levels of lecithin cholesterol acyltransferase and cardiovascular risk in postmenopausal women.

(c) 2019 Sociedad Colombiana de Cardiología y Cirugía Cardiovascular. Published by Elsevier España, S.L.U. This is an open access article under the CC BY-NC-ND license (http:// creativecommons.org/licenses/by-nc-nd/4.0/).
\end{abstract}

\section{Introducción}

En el 2012 las enfermedades no transmisibles fueron responsables del $68 \%$ de las defunciones en el mundo y del $52 \%$ de las consideradas muertes prematuras (muertes de personas menores de 70 años); cerca del $46 \%$ de esas muertes (17,5 millones) están asociadas a enfermedades cardiovasculares $^{1}$. Desde el punto de vista fisiológico y metabólico, las principales alteraciones asociadas a riesgo cardiovascular son la hipertensión arterial, la obesidad, la hiperglicemia y la hiperlipidemia ${ }^{2}$. La mujer postmenopáusica es especialmente vulnerable a las enfermedades cardiovasculares debido a la disminución de los niveles de estrógenos producto de la falla ovárica, la cual produce, a corto plazo, signos y síntomas en los sistemas vasomotor, neuropsiquiátrico, genitourinario, cardiovascular y osteomuscular $^{2}$, que, asociados a un estilo de vida inadecuado, generan aumento de la grasa visceral, resistencia a la insulina e intolerancia a la glucosa, dislipidemias y elevación de la presión arterial, alteraciones que se relacionan directamente con el aumento en el riesgo de enfermedades cardiovasculares ${ }^{3}$.

Las altas concentraciones lipémicas guardan una relación proporcional directa con el desarrollo de enfermedades cardiovasculares, ya que cuanto mayor sea el nivel de lípidos en sangre de forma crónica, mayor es la probabilidad de padecer disfunción del endotelio vascular coronario como producto de la formación de la placa ateromatosa y la respuesta inflamatoria subsiguiente. No obstante, aún se desconocen muchos de los mecanismos que intervienen en la formación del ateroma y el papel fundamental de algunas partículas lipémicas circulantes en el riesgo cardiovascular ${ }^{4,5}$. En la actualidad se sabe que la enzima lecitina colesterol aciltransferasa tiene un papel clave en el metabolismo de las lipoproteínas de alta densidad (HDL) y que su deficiencia se relaciona con la reducción de las concentraciones séricas de HDL, pero no se ha dilucidado por completo su rol en la formación de la placa ateromatosa, posiblemente porque los niveles bajos de $\mathrm{HDL}$ asociados a alteraciones genéticas de la enzima en mención no se asocian a riesgo cardiovascular, mientras que los niveles bajos de HDL por otras causas sí se relacionan directamente con riesgo cardiovascular ${ }^{6}$.

La lecitina colesterol aciltransferasa es una enzima lipoproteica cuya función es la de esterificar el colesterol libre extracelular para ser transportado por las HDL al hígado y otros tejidos, manteniendo el equilibrio entre las concentraciones plasmáticas y tisulares de colesterol libre. La deficiencia de lecitina colesterol aciltransferasa por causas genéticas es muy poco frecuente y se asocia con alteraciones de la córnea, los riñones y los eritrocitos debido a la acumulación de colesterol no esterificado. Aunque estos pacientes tienen una deficiencia severa de HDL, esta no se ha asociado a aterosclerosis ${ }^{7}$.

Son muchos aun los aspectos relacionados con la lecitina colesterol aciltransferasa y su relación con el riesgo cardiovascular que necesitan ser dilucidados. Adicionalmente, existen pocos estudios que evalúan los valores séricos de esta enzima y su relación con variables bioquímicas y antropométricas asociadas a riesgo cardiovascular y casi ninguno en mujeres postmenopáusicas de la Región Caribe. En este trabajo de investigación se determinaron los valores séricos 
de lecitina colesterol aciltransferasa en un grupo de mujeres postmenopáusicas trabajadoras de una institución de salud de Barranquilla y se estableció su relación con factores de riesgo cardiovascular, como dislipidemia, hiperglicemia, perímetro abdominal e índice de masa corporal con el fin de aportar evidencia científica que a futuro impacte en los criterios diagnósticos y terapéuticos en este grupo poblacional.

\section{Materiales y métodos}

En este estudio descriptivo transversal, correlacional, se incluyeron 56 mujeres postmenopáusicas (un año posterior a la última menstruación) entre 50 y 65 años de edad. Para ello se realizó un muestreo de oportunidad entre las trabajadoras de una institución de salud, teniendo en cuenta criterios de inclusión, como no estar consumiendo medicación para el control de hiperglicemia, dislipidemia o usar tratamiento para reposición hormonal. Posterior a la firma del consentimiento informado, las mujeres fueron citadas en las horas de la mañana para una extracción de muestra sanguínea, previo ayuno de 12 horas; la última ingestión de alimentos debía ser una comida libre de grasa. Las muestras de sangre de $7 \mathrm{ml}$ fueron extraídas en tubo sin anticoagulante (sistema vacutainer) por una bacterióloga experta; posteriormente se centrifugaron para la obtención del suero y la realización del perfil lipídico y la glicemia en el laboratorio clínico de la Fundación Hospital Universitario Metropolitano (FHUM).

Alícuotas de suero de más de $0,5 \mathrm{ml}$ se almacenaron a $-80^{\circ} \mathrm{C}$ para la posterior determinación sérica de lecitina colesterol aciltransferasa mediante el kit comercial lecithin cholesterol acyltransferase (lecitina colesterol aciltransferasa) ELISA kit de la casa comercial Cell Biolabs, cuyo fundamento analítico es un ELISA competitivo. Los procedimientos se llevaron a cabo de acuerdo con las instrucciones del fabricante y el uso del equipo Stat Fax 4200 Awareness Technology en el laboratorio clínico de la FHUM. La valoración antropométrica de las mujeres estuvo a cargo de una nutricionista experta, quien usó estadiómetro, balanza digital y cinta métrica marca SECA, de acuerdo con los protocolos establecidos en la resolución 2465 del año $2016^{8}$. Los resultados de los análisis bioquímicos, glicemia basal y perfil lipídico se entregaron y socializaron con las participantes, para que de ser necesario se remitieran a su Entidad Prestadora de Salud para posible tratamiento farmacológico y seguimiento bajo control médico.

Se calculó la frecuencia de mujeres que para cada variable mostraban alteraciones consideraras como factor de riesgo cardiovascular según la Asociación Latinoamericana de Diabetes -ALAD- y los criterios armonizados por la Organización Mundial de la Salud (OMS) y la Federación Internacional de Diabetes, entre otras entidades internacionales (criterios APT III), así: obesidad abdominal demostrada con un perímetro abdominal $\geq 88 \mathrm{~cm}$, triglicéridos $>150 \mathrm{mg} / \mathrm{dl}$, colesterol $\mathrm{HDL}<50 \mathrm{mg} / \mathrm{dl}$ y glicemia anormal en ayunas (superior a $110 \mathrm{mg} / \mathrm{dl}$ ). Aunque los valores séricos de $L D L>140 \mathrm{mg} / \mathrm{dl}$ no están contemplados dentro de los criterios NCEP-ATP III son considerados un importante factor de riesgo de enfermedades cardiovasculares ${ }^{9-11}$.

Mediante el programa estadístico Statgraphics Plus Professional 16.0 .03 se establecieron relaciones entre los niveles séricos de lecitina colesterol aciltransferasa y las variables bioquímicas y antropométricas a través de un análisis de regresión simple. Se compararon también los valores promedio de lecitina colesterol aciltransferasa entre los grupos, que para cada variable se encontraban en condición o no de riesgo cardiovascular can base en los criterios ATPIII (test de ANOVA).

Este proyecto fue aprobado por el comité de ética en investigación de la Universidad Metropolitana, acorde con lo establecido en la resolución 8430 de 1993 del Ministerio de Salud de Colombia y los principios éticos para las investigaciones establecidos en la Declaración de Helsinki.

\section{Resultados}

Cincuenta y seis mujeres postmenopáusicas con edades promedio de 55,89 $\pm 5,74$ años (rango 50-65), vinculadas al estudio fueron evaluadas en cuanto a antropometría y se les realizaron análisis bioquímicos (glicemia basal, perfil lipídico y valores séricos de lecitina colesterol aciltransferasa). Los valores promedio de los resultados de las variables antropométricas fueron $70,08 \pm 11,41 \mathrm{~kg}$ para el peso, $157 \pm 6,8 \mathrm{~cm}$ para la talla y $28,12 \pm 3,91$ para el índice de masa corporal. Teniendo en cuenta los criterios de la OMS para la clasificación nutricional por antropometría en la población adulta ${ }^{8}$, el $17,85 \%(n=10)$ de las mujeres tenía índice de masa corporal normal (menor de 25 ), el 53,57\% $(n=30)$ se encontraba con sobrepeso (índice de masa corporal igual o superior a 25$)$ y el $28,57 \%(n=16)$ tenía obesidad (índice de masa corporal superior a 30). Respecto al perímetro abdominal el $82,14 \%$ de las mujeres $(n=46)$ tenía una medida superior a $88 \mathrm{~cm}$, considerada de acuerdo con los criterios del NCEP-ATP III como factor de riesgo cardiovascular.

Los valores séricos promedio de lecitina colesterol aciltransferasa fueron 7,89 $\pm 1,26 \mu \mathrm{g} / \mathrm{ml}$. La tabla 1 muestra el promedio de los valores de las variables bioquímicas y antropométricas en la población total analizada, y el porcentaje de mujeres que, de acuerdo con los criterios del NCEPATP III, tienen valores alterados de las variables asociados a riesgo cardiovascular y los valores promedio de lecitina colesterol aciltransferasa en relación con dicha condición para cada variable.

El análisis de regresión lineal al comparar la variable lecitina colesterol aciltransferasa sérica en $\mu \mathrm{g} / \mathrm{ml}$, con cada una de las variables estudiadas (fig. 1), muestra que las variables que tienen mayor relación lineal con los valores de lecitina colesterol aciltransferasa son el índice de masa corporal y el perímetro abdominal; sin embargo, esta relación no es estadísticamente significativa $(p>0,05)$.

\section{Discusión}

En mujeres postmenopáusicas las enfermedades cardiovasculares son una de las causas más importantes de morbimortalidad. Los cambios físicos y metabólicos mediados por la alteración hormonal, asociados a cambios en el estilo de vida, conllevan aumento del índice de masa corporal y alteraciones bioquímicas, que en muchas ocasiones generan incremento del riesgo de padecer enfermedades cardiovasculares ${ }^{12}$. La enzima lecitina colesterol 
Tabla 1 Promedio de los valores para cada variable analizada y de lecitina colesterol aciltransferasa en el grupo de mujeres con alteraciones en las variables asociadas a riesgo cardiovascular

\begin{tabular}{|c|c|c|c|c|c|c|}
\hline \multirow[t]{2}{*}{ Variables } & \multirow{2}{*}{$\begin{array}{l}\text { Población total } \\
(n=56)\end{array}$} & \multicolumn{2}{|l|}{ Riesgo } & \multicolumn{2}{|l|}{ No riesgo } & \multirow[t]{2}{*}{$\mathrm{P} 2$} \\
\hline & & n (\%) & $\begin{array}{l}\text { Lecitina } \\
\text { colesterol } \\
\text { aciltransferasa } \\
\mu \mathrm{g} / \mathrm{ml}\end{array}$ & n (\%) & $\begin{array}{l}\text { Lecitina } \\
\text { colesterol } \\
\text { aciltransferasa } \\
\mu \mathrm{g} / \mathrm{ml}\end{array}$ & \\
\hline $\begin{array}{l}\text { Lecitina colesterol } \\
\text { aciltransferasa }(\mu \mathrm{g} / \mathrm{ml})\end{array}$ & $7,89 \pm 1,26$ & & & & & \\
\hline Índice de masa corporal & $28,12 \pm 3,91$ & $46(82,14)$ & $8,06 \pm 1,25$ & $10(17,85)$ & $7,12 \pm 1,21$ & 0,032 \\
\hline $\begin{array}{l}\text { Perímetro abdominal } \\
(\mathrm{cm})\end{array}$ & $95,80 \pm 10,30$ & $46(82,14)$ & $8,03 \pm 1,18$ & $10(17,85)$ & $7,25 \pm 1,49$ & 0,079 \\
\hline Glicemia (mg/dl) & $96,57 \pm 11,97$ & $11(19,64)$ & $7,80 \pm 1,45$ & $45(80,35)$ & $7,91 \pm 1,23$ & 0,793 \\
\hline Colesterol total $(\mathrm{mg} / \mathrm{dl})$ & $216,18 \pm 40,28$ & $35(62,50)$ & $8,00 \pm 1,21$ & $21(37,50)$ & $7,71 \pm 0,36$ & 0,207 \\
\hline Triglicéridos (mg/dl) & $149,60 \pm 72,54$ & $22(39,28)$ & $7,80 \pm 1,21$ & $34(60,71)$ & $7,95 \pm 1,3$ & 0,669 \\
\hline $\mathrm{HDL}(\mathrm{mg} / \mathrm{dl})$ & $45,78 \pm 8,77$ & $40(71,42)$ & $7,86 \pm 1,25$ & $16(28,57)$ & $7,97 \pm 1,32$ & 0,773 \\
\hline LDL (mg/dl) & $140,13 \pm 35,76$ & $31(55,35)$ & $8,04 \pm 1,21$ & $25(44,64)$ & $7,71 \pm 1,32$ & 0,339 \\
\hline
\end{tabular}

Muestra el promedio \pm desviación estándar (DS) de los valores para cada variable analizada en la población total. Valores promedio \pm desviación estándar del valor de lecitina colesterol aciltransferasa sérica para la población (n (\%)) en cada variable de acuerdo con la condición o no de riesgo (criterios ATP III): Perímetro abdominal $\geq 88 \mathrm{~cm}$, glicemia $>110 \mathrm{mg} / \mathrm{dl}$, colesterol total $>200 \mathrm{mg} / \mathrm{dl}$, triglicéridos $>150 \mathrm{mg} / \mathrm{dl}, \mathrm{HDL}<50 \mathrm{mg} / \mathrm{dl}$ y $\mathrm{LDL}>140 \mathrm{mg} / \mathrm{dl}$ y para la presencia de sobrepeso u obesidad (índice de masa corporal $\geq 25$ ). El valor de la significancia estadística $(P)$ al comparar los valores promedio de lecitina colesterol aciltransferasa de acuerdo con el nivel de riesgo para cada variable (Test de ANOVA), es representativo cuando es inferior a 0,05. HDL: lipoproteína de alta densidad; LDL: lipoproteína de baja densidad; VLDL: lipoproteína de muy baja densidad.

aciltransferasa promueve la transferencia del colesterol libre desde los tejidos hasta el hígado para ser excretado, manteniendo las concentraciones plasmáticas y tisulares de colesterol libre en equilibrio, ṕero aún no se ha dilucidado por completo el papel de la lecitina colesterol aciltransferasa en la patogenia de la aterosclerosis humana ${ }^{13}$.

En este estudio, el análisis de regresión lineal no mostró relaciones significativas entre los valores séricos de lecitina colesterol aciltransferasa y las variables bioquímicas y antropométricas analizadas; sin embargo, al comparar los valores promedio de lecitina colesterol aciltransferasa entre las mujeres con índice de masa corporal normal y las clasificadas en sobrepeso u obesidad, se encontró que las cifras de lecitina colesterol aciltransferasa en las mujeres con índice de masa corporal inferior a 25 eran significativamente mayores, tendencia observada también en las mujeres con perímetro abdominal superior a $88 \mathrm{~cm}$, aunque no de manera estadísticamente significativa. Los resultados de estudios previos reportados por otros autores, son contradictorios a la hora de definir el papel de la lecitina colesterol aciltransferasa en la aterosclerosis específicamente relacionado con el riesgo de presentar en el futuro un evento vascular. Al respecto, Holleboom et al. en un estudio de casos y controles, no encontraron diferencias significativas en la concentración y actividad de la lecitina colesterol aciltransferasa entre pacientes con enfermedades cardiovasculares y sanos, pero sí determinaron que la enzima está más elevada en las mujeres que en los hombres y que este incremento se asocia con aumento en el riesgo de padecer enfermedad vascular que no fue explicado por la disminución del HDL ya que inesperadamente no se encontró relación entre ambos indicadores $^{13}$.
Vale destacar que estudios previos de otros autores demuestran una relación adecuada entre los valores séricos de lecitina colesterol aciltransferasa y su actividad enzimática ${ }^{14}$; sin embargo, nuevos descubrimientos con relación a la complejidad en la estructura y función de esta enzima, así como la variedad de mutaciones que pueden modificar su actividad hacen posible que las concentraciones séricas de lecitina colesterol aciltransferasa evaluadas en este estudio y la actividad enzimática no sean del todo equivalentes ${ }^{15,16}$, más aun cuando en el contexto regional en el que se realizó este estudio no se han reportado estudios relativos a los genes que codifican para esta enzima, desconociendo la posible existencia de variables con mayor o menor actividad.

Aunque los resultados de este estudio no permiten proponer los valores séricos de lecitina colesterol aciltransferasa como un marcador de riesgo cardiovascular, estudios como el de Rhainds et al. plantean esta posibilidad, ya que determinaron que los pacientes con ateroma coronario diagnosticado mediante ultrasonido intravascular tienen valores de lecitina colesterol aciltransferasa séricos superiores $(8,94 \pm 2,51 \mu \mathrm{g} / \mathrm{ml})$ con relación a un grupo control de pacientes sanos $(7,89 \pm 2,99 \mu \mathrm{g} / \mathrm{ml})$, y que esos valores se correlacionaban de manera positiva con LDL y triglicéridos, hecho que sugiere que la prueba con lecitina colesterol aciltransferasa sería válida como factor predictor de enfermedad coronaria y podría tenerse en cuenta a futuro como un posible blanco terapéutico ${ }^{17}$.

En la actualidad, diversos autores debaten el papel protector contra la arterioesclerosis atribuido a los niveles séricos elevados de HDL; más contradictorios aún son los estudios que tratan de definir la influencia de la lecitina colesterol aciltransferasa en el riesgo cardiovascular. En una 

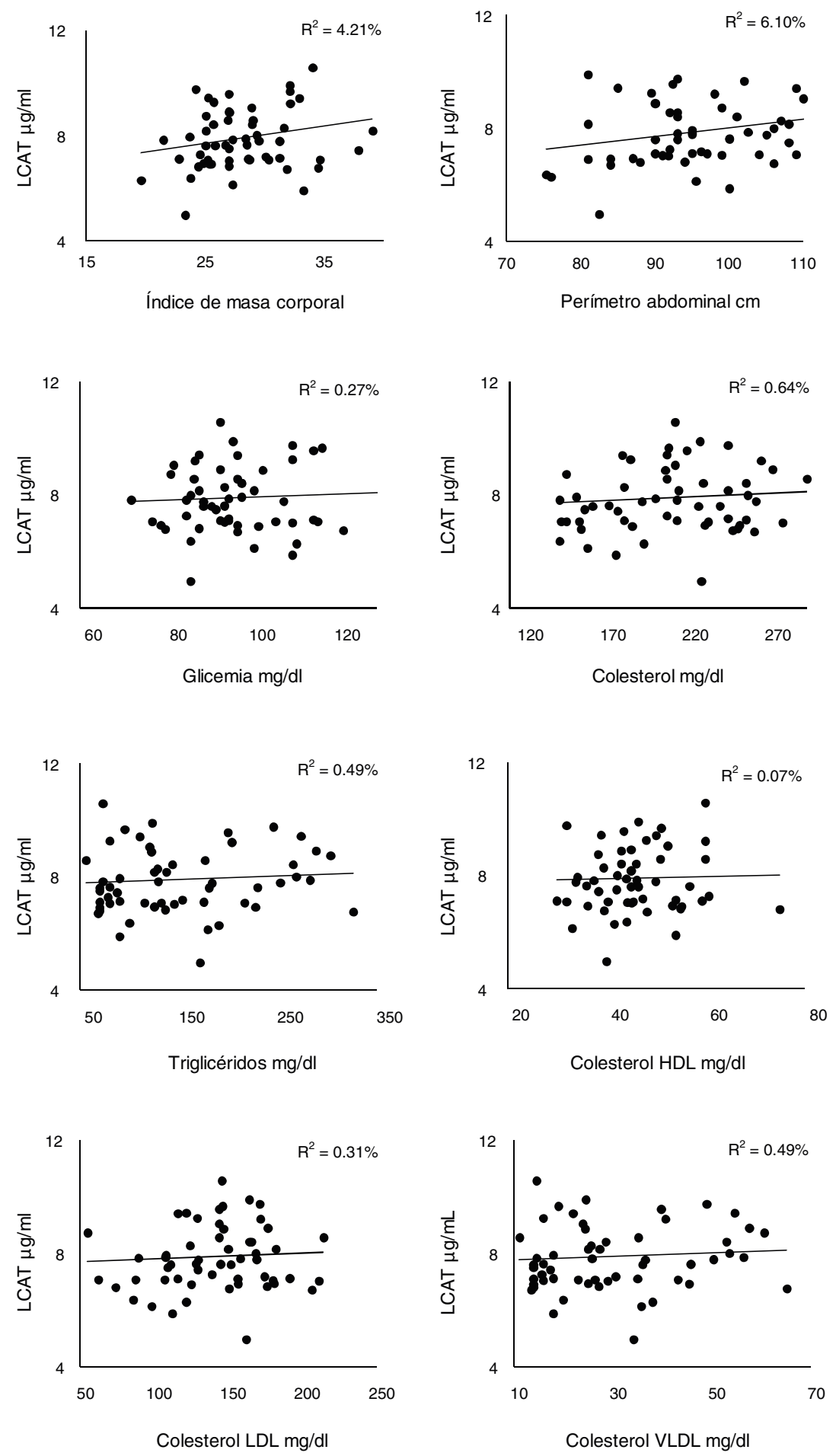

Figura 1 Diagramas de dispersión que muestran la relación entre los valores de lecitina colesterol aciltransferasa sérica en $\mu g / \mathrm{ml}$ y las variables bioquímicas y antropométricas analizadas. $\mathrm{R}^{2}$ : coeficiente de determinación expresado en porcentaje. El valor de significancia estadística $(p)$ del análisis de la varianza (ANOVA) de la regresión lineal es significativo si $p<0,05$. HDL: lipoproteína de alta densidad; LDL: lipoproteína de baja densidad; VLDL: lipoproteína de muy baja densidad.

revisión de tema, Vergeer et al. sintetizan los hallazgos de estudios en animales transgénicos que han tratado de definir dicho efecto pero no han tenido hallazgos concluyentes ${ }^{18}$. Aunque los estudios en humanos tampoco han podido definir el papel de la lecitina colesterol aciltransferasa en el riesgo cardiovascular, vale destacar que el EPIC Norfolk (que incluyó 25.000 hombres y mujeres entre los 40-79 años de edad durante los años 1993 y 1997) y el IMPROVE (que incluyó 540 adultos asintomáticos pero con factores de riesgo cardiovascular) coinciden en reportar una 
relación directamente proporcional entre los niveles séricos de lecitina colesterol aciltransferasa y factores de riesgo cardiovascular en mujeres ${ }^{18}$.

En el presente estudio los valores séricos promedio de lecitina colesterol aciltransferasa fueron 7,89 $\pm 1,26 \mu \mathrm{g} / \mathrm{ml}$; lamentablemente no hay un referente regional de los valores séricos de dicha enzima en la población adulta sin factores de riesgo asociados o estudios previos en mujeres postmenopáusicas en el Caribe colombiano. Sin embargo, los valores encontrados resultan superiores a los publicados por Albers et al. quienes reportaron en una población en edades comprendidas desde los 20 y hasta los 59 años, valores parcialmente más altos en mujeres $(5,90 \pm 1,06$, $\mathrm{n}=154)$ comparado con hombres $(5,49 \pm 0,89, \mathrm{n}=83)$, utilizando la técnica de radioinmunoensayo; determinaron, además, que los valores de lecitina colesterol aciltransferasa fueron correlacionados positivamente con el índice de masa corporal, colesterol total y colesterol LDL ${ }^{19}$.

La relación entre obesidad y valores séricos elevados de lecitina colesterol aciltransferasa como los encontrados en este estudio, fueron reportados previamente por Asztalos et al. al evaluar 19 mujeres con obesidad mórbida antes y después de realizada una cirugía de bypass gástrico, observando que 12 meses posterior a la cirugía los valores séricos de esta enzima descendieron de $9,3 \pm 2,1 \mu \mathrm{g} / \mathrm{ml}$ a $7,4 \pm 1,6 \mu \mathrm{g} / \mathrm{ml}$ y que ese descenso era coherente con el comportamiento del índice de masa corporal, el colesterol LDL y los niveles plasmáticos de insulina en las pacientes ${ }^{20}$.

\section{Conclusiones}

Este trabajo constituye uno de los primeros que evalúa los niveles séricos de lecitina colesterol aciltransferasa en mujeres post menopaúsicas del Caribe Colombiano y aunque no se lograron establecer mediante análisis de regresión lineal relaciones significativas entre los valores séricos de lecitina colesterol aciltransferasa y los factores bioquímicos y antropométricos asociados a riesgo cardiovascular analizados, sí se encontró una relación directa significativa al comparar los promedios de los valores séricos de lecitina colesterol aciltransferasa entre las mujeres con índice de masa corporal normal y las que lo tenían aumentado, coherente con lo reportado en otros estudios. Resulta necesario y pertinente plantear nuevas investigaciones que contribuyan al esclarecimiento de las implicaciones de la lecitina colesterol aciltransferasa en el riesgo cardiovascular de este grupo poblacional en el contexto regional, teniendo en cuenta que las enfermedades no transmisibles, y entre estas las enfermedades cardiovasculares, tienen actualmente un importante impacto en los indicadores de morbilidad y mortalidad en esta población.

\section{Financiación}

Ninguna.

\section{Conflicto de intereses}

Ninguno.

\section{Agradecimientos}

A la Universidad Metropolitana y a la Fundación Hospital Universitario Metropolitano.

\section{Bibliografía}

1. World health organization. Informe sobre la situación mundial de las enfermedades no transmisibles. 2014. [internet]. Disponible en: http://apps.who.int/iris/ bitstream/handle/10665/149296/WHO_NMH_NVI_15.1_spa.pdf

2. Kunstmann S, De grazia R, Gainza D. Aterosclerosis en la mujer: factores de riesgo y prevención. Revista Chilena de Cardiología. 2012;31:142-7.

3. Buckley N. Meta-analysis?: Effect of hormone-replacement therapy on components of the metabolic syndrome in postmenopausal women. Diabetes Obes Metab. 2006;8:538-54.

4. Bahrami M, Barati H, Mehdi M, Golmohammadi T. Lipoprotein lipase gene variants?: Association with acute myocardial infarction and lipid profiles. Egypt J Med Hum Genet. 2015;16: 327-32.

5. Rosenson RS, Davidson MH, Hirsh BJ, Kathiresan S, Gaudet D. Genetics and Causality of Triglyceride-Rich Lipoproteins in Atherosclerotic Cardiovascular Disease. J Am Coll Cardiol. 2014;64.23:2525-40.

6. Haase CL, Tybjærg-hansen A, Qayyum AA, Schou J, Nordestgaard BG, Frikke-schmidt R, Disease:. A Mendelian Randomization Study of HDL Cholesterol in 54,500 Individuals. 2012;97:248-56.

7. Lucca F, Ossoli A, Boscutti G, Franceschini G, Calabresi L. Lecithin: cholesterol acyltransferase deficiency, from genes to therapy. Giornale italiano di nefrologia: organo ufficiale della Societa italiana di nefrologia. 2016;33:S68.

8. Ministerio de Salud y Protección social de Colombia. Manual de Implementación de la resolución 2465 de 2016. 2016. Disponible en: http://www.saludcapital.gov.co/ Su_GPAISP/Caja_de_herramientas/2_DOCTO_DE_CONSULTA/SAN/ DESNUTRICI\%C3\%93N/1_Patrones_Referencia/3_Man_Imp_Res_ 2465.pdf

9. Lizazaburu Robles JC. Síndrome metabólico?: concepto y aplicación práctica Metabolic syndrome?: concept and practical application. An Fac Med. 2013;74:315-20.

10. Expert Panel on Detection, Evaluation, and Treatment of High Blood Cholesterol in Adults (Adult Treatment Panel III). Third Report of the National Cholesterol Education Program (NCEP) Final Report. Circulation. 2002; 106:3143.

11. Miccoli R, Pisa U, Bianchi C. Prevalence of the metabolic syndrome among Italian adults according to ATP III definition. Nutrition, metabolism and cardiovascular diseases. 2005; 15:250-4

12. Villaverde Gutiérrez C, Ramírez Rodrigo J, Olmedo Alguacil MM, Sánchez Caravaca MÁ, Argente del Castillo Lechuga MJ, Ruiz Villaverde A. Overweight obesity and cardiovascular risk in menopausal transition. Nutrición Hospitalaria. 2015;32: 1603-8.

13. Holleboom AG, Kuivenhoven JA, Vergeer M. Plasma levels of lecithin?: cholesterol acyltransferase and risk of future coronary artery disease in apparently healthy men and women?: a prospective case-control analysis nested in the EPIC-Norfolk population study. J Lipid Research. 2010;51:416-21.

14. Kobori K, Saito K, Ito S, Kotani K, Manabe M, Kanno T. A new enzyme-linked immunosorbent assay with two monoclonal antibodies to specific epitopes measures human lecithin-cholesterol acyltransferase. J Lipid Research. 2002;43:325-34.

15. Gu X, Wu Z, Huang Y. A Systematic Investigation of Structure /Function Requirements for the Apolipoprotein A-I 
/Lecithin Cholesterol Acyltransferase Interaction Loop ofHighdensity. Journal of Biological Chemistry. 2016;291:6386-95, http://dx.doi.org/10.1074/jbc.M115.696088.

16. Glukhova A, Hinkovska-galcheva V, Kelly R. Structure and function of lysosomal phospholipase A2 and lecithin: cholesterol acyltransferase. Nature Communications. 2015;6:6250.

17. Rhainds D, Gebhard C, Cossette M, Guertin MC, Rhéaume E, Tardif J. C. Elevated levels of lecithin: cholesterol acyltransferase (lecitina colesterol aciltransferasa) are associated with reduced atheroma burden-An ivus study. Atherosclerosis. Nature Communications. 2016;252:e106.
18. Vergeer M, Holleboom AG, Kastelein JJP, Kuivenhoven JA, The HDL. hypothesis?: does high-density lipoprotein protect from atherosclerosis?? Journal Lipid Research. 2010;51: 2058-73.

19. Albers JJ, Bergelin RO, Adolphson JL, Wahl PW. Populationbased reference values for lecithin-cholesterol acyltransferase (lecitina colesterol aciltransferasa). Atherosclerosis. 1982;43(2-3):369-79.

20. Asztalos BF, Swarbrick MM, Schaefer EJ. Effects of weight loss, induced by gastric bypass surgery, on HDL remodeling in obese women. Journal Lipid Research. 2010;51:2405-12. 